\title{
Surgical Management of Periorbital Squamous Cell Carcinoma through Wide Margin Excision Followed by Transposition Skin Flap in a Dog
}

\author{
M. Gokulakrishnan ${ }^{1 *}$, L. Nagarajan ${ }^{2}$, Ramani $^{2}$ and Sowmiya ${ }^{2}$ \\ ${ }^{1}$ Department of Clinics, Madras Veterinary College, Chennai, India \\ ${ }^{2}$ Department of Veterinary Surgery and Radiology, Madras Veterinary College, Tamil Nadu \\ Veterinary and Animal Sciences University, Chennai-600007, India \\ *Corresponding author
}

\section{A B S T R A C T}

\section{Keywords \\ Squamous Cell Carcinoma - Wide margin excision - Transposition flap- Dog.}

Article Info

Accepted:

18 January 2020 Available Online: 10 February 2020
A8--year-old intact male dog was brought to Madras Veterinary College Teaching Hospital with a history of progressive ulcerative mass at the left periorbital area for the past 3 months. Haemato-biochemical analysis was done, which revealed thrombocytopenia, neutrophilia shift to left and a marginal increase in ALP levels. Absence of metastasis and bony involvement were observed on plain radiograph. Corneal swab and a fine needle biopsy were done to rule out ophthalmic health and nature of tumor cells if any, which revealed squamous cell carcinoma. Since the mass was extensive routine excision would result in a wide defect to be opposed by standard suturing technique hence a transposition skin flap was performed. The animal had an uneventful recovery.

\section{Introduction}

Squamous cell carcinoma (SCC) is a malignant neoplasm arising from squamous epithelium. Squamous cell carcinoma (SCC) is a relatively common, malignant neoplasm of dogs and cats that can arise in a variety of locations. SCCs account for $15 \%$ of skin tumors in cats. In dogs, less than $5 \%$ of cutaneous neoplasms are SCC, and common sites include the legs, scrotum, perineum, nasal planum, and various lightly pigmented areas. O'Brien et al., (2016) SCCs account for $70 \%$ of feline and $25 \%$ of canine oral neoplasms and may arise from virtually any surface in the oral cavity, including the gingival, tongue, tonsils, pharynx, lips, and buccal mucosa. Schmidt et al., (2001) Several treatment modalities exist, but surgical excision, if possible, is regarded as the best treatment option. Ward et al., (2007) Early diagnosis and treatment of SCC are key because small, early-stage tumors are the most amenable to treatment and carry the best 
prognosis. Surgical excision is the primary treatment option for most patients with SCC. The ability to completely excise the tumor depends on factors such as the size and location of the tumor.

A 8-year-old intact male spitz dog weighing around $6 \mathrm{kgs}$ was presented to Madras Veterinary College Teaching Hospital, small animal outpatient unit for evaluation and treatment with a complaint of progressive ulcerative mass at the left periorbital area for the past 3 months. On clinical examination, the periorbital area was hemorrhagic, ulcerative and non-painful. Ophthalmic examination revealed any abnormality in the affected eye with no abnormalities in the vision.

Fine needle aspiration cytology was performed to rule out the nature of cells. A corneal swab was performed to rule out the nature of cells. And any metastasis of the periorbital lesion into the eye. FNAC revealed squamous cell carcinoma of the left periorbital area with noticeable numerous neutrophils and few clusters of pleomorphic squamous epithelial cells. Routine hemato biochemical profile was taken to diagnose general organ health and any abnormalities, which revealed mild anemia, neutrophilia with shift to left, thrombocytopaenia and elevated alp levels with altered calcium phosphorous ratio.

Lateral thoracic and skull radiographs were taken to rule out any metastasis and bony involvement that reveaked any abnormality. Appropriate hematonics and platelet enrich mane supplements were advised for a week. Since the squamous cell carcinoma was extensive, wide margin excision was performed following which a broad defect was observed.

A subconjunctival flap was performed to maintain the health of the eye. Following which a transposition flap was performed. Appropriate dressings and post-operative care were administered until the dog had an uneventful recovery. The pet was premedicated with Butorphanol and Diazepam at the dose rate of 0.25 and $0.1 \mathrm{mg} / \mathrm{kg}$ body weight intravenously. The animal was induced anaesthesia with propofol at the dose rate of $4 \mathrm{mg} / \mathrm{kg}$ intravenous and anaesthesia was maintained with isoflurane with oxygen as a carrier gas under rebreathing circuit.

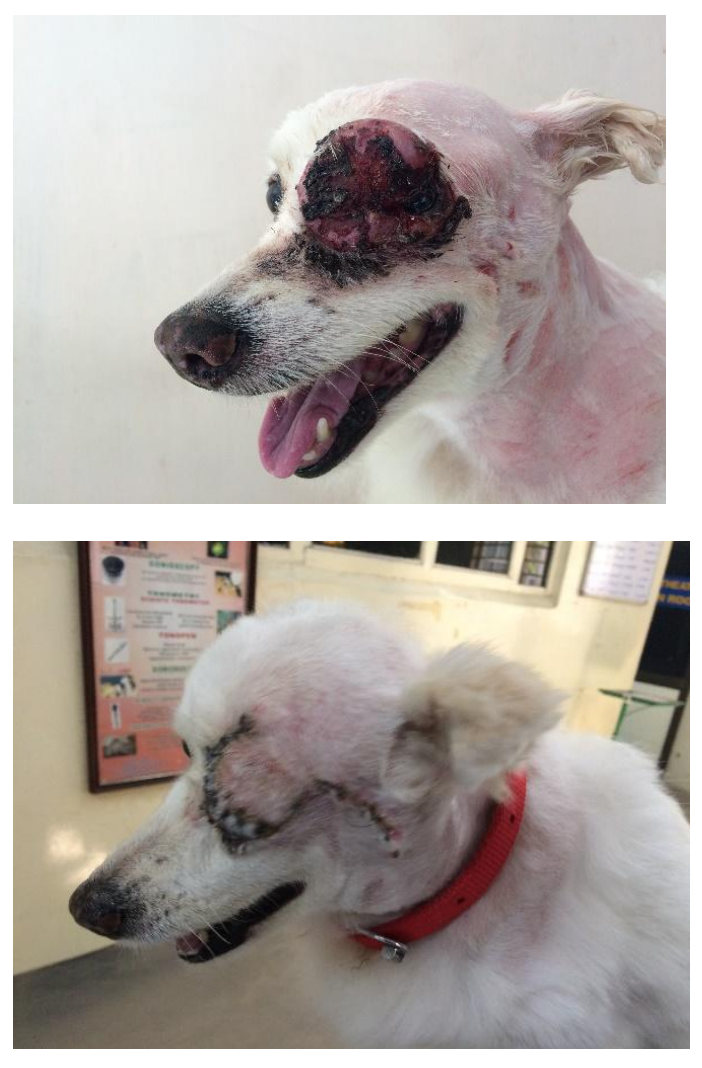

After surgical preparation of the pet, the periorbital area was prepared, wide surgical margination was marked with a felt pen marker and the squamous cell carcinoma was excised after proper ligation with thermocautery and $\mathrm{CO}_{2}$ diode laser. Sub conjunctival flap was performed following which the surgical wound created after excision that was inappropriate to be opposed by standard suturing method was therefore performed transposition skin flap after delineation of the flap margins. 
Postoperative antibiotics and antiinflammatory drugs with appropriate dressing on alterative days were done. The pet had an uneventful recovery after 21 days.

Squamous cell carcinoma (SCC) is a relatively common, malignant neoplasm of dogs and cats that can arise in a variety of locations. Bacon et al., (2007) Several treatment modalities exist, but surgical excision, if possible, is regarded as the best treatment option. Boria et al., (2004)

Early diagnosis and treatment of SCC are key because small, early-stage tumors are the most amenable to treatment and carry the best prognosis. Brooks et al., (2018) Since the tumor was massive wide margination excision was planned. Older animals are at greater risk for developing SCC, with the average age at presentation being 8 to 10 years for dogs as seen in the present case and 10 to 12 years for cats. Coindre et al., (2001) Prolonged exposure to UV light, lack of skin pigment, and a sparse haircoat all contribute to the development of cutaneous SCC. Connery et $a l$. , and Dennis et al., (2006) Dogs with white haircoats are more susceptible to cutaneous SCC as in the present case whereas dogs with dark haircoats appear to be overrepresented in cases of digital tumors. Forrest et al., (2000) Rarely has a sex predilection been reported; in one study, female dogs appeared to be at increased risk for development of lingual $\mathrm{SCC}^{4}$, while tonsillar SCC may be more common in male dogs. Lascelles et al., (2001)

Early diagnosis of SCC is paramount for early therapeutic intervention, which may result in long-term control or cure for affected patients. Latimer et al., (2001) SCC may be suspected based on the gross appearance of a lesion and its location, but definitive diagnosis requires microscopic examination of the affected tissue. Cytology is a rapid, easy, non-invasive method that may provide the diagnosis of SCC and is often attempted as the first diagnostic technique, especially for cutaneous lesions as performed in the present case. Biopsy with histopathology may be required to obtain a definitive diagnosis if cytology is non-diagnostic or equivocal. SCCs generally are not considered chemo responsive tumors; however, chemotherapy may be considered under certain circumstances. Lieb and $\mathrm{Li}$ (2006) For example, chemotherapy may be advised for tumors that are inoperable, anaplastic, or metastatic at the time of diagnosis, in the present case since the tumor was not metastatic and had absence of bony involvement surgical excision was therefore performed. McEntee et al., (2004)

Since the post-operative defect was not opposed by standard suturing technique transposition flap was performed. The transposition $\mathrm{fl}$ ap is a local flap technique with a wide variety of uses. It is a rotating flap that is applicable in most body regions in the dog, cat, and other species, including birds. When it is based on the sub dermal plexus as its primary source of circulation, its size is more limited than axial pattern flaps Porat et $a l$. , (2008). The survival of sub dermal plexus flap in the above case was $98 \%$ which was measured subjectively as per observations of Plassmann (2005).

\section{References}

Bacon N.J., Dernell W.S., Ehrhart N., Powers B.E., Withrow S.J. (2007). Evaluation of primary re-excision after recent inadequate resection of soft tissue sarcomas in dogs: 41 cases (19992004). Journal of the American Veterinary Medical Association 230, 548-555.

Boria P.A., Murray D.J., Bennett P.F, et al., (2004) Evaluation of cisplatin combined with piroxicam for the treatment of oral 
malignant melanoma and oral squamous cell carcinoma in dogs. JAVMA ;224(3):388-394.

Brooks M.B., Matus R.E andC.E.Leifer, et al., (2018) Chemotherapy versus chemotherapy plus radiotherapy in the treatment of tonsillar squamous cell carcinoma in the dog. J Vet Intern Med ;2:206-211.

Coindre J.M., Terrier P., Guillou L., Le Doussal V., Collin F., Ranchère D., Sastre X., Vilain M.O., Bonichon F., N'Guyen Bui B. (2001). Predictive value of grade for metastasis development in the main histologic types of adult soft tissue sarcomas: a study of 1240 patients from the French Federation of Cancer Centers Sarcoma Group. Cancer 91, 1914-1926.

Connery N.A., Bellenger C.R. (2002). Surgical management of haemangiopericytoma involving the biceps femoris muscle in four dogs. Journal of Small Animal Practice 43, 497-500.

Dennis M.M., Ehrhart N, Duncan C.G., et al., (2006) Frequency of and risk factors associated with lingual lesions in dogs: 1,196 cases (19952004). JAVMA ;228(10):1533-1537.

Forrest L.J., Chun R., Adams W.M., Cooley A.J., Vail D.M. (2000). Postoperative radiotherapy for canine soft

Lascelles B.D., Parry A.T., Stidworthy M.F, et al., (2000) Squamous cell carcinoma of the nasal planum in 17 dogs. Vet Rec ;147:473-476.
Latimer K.S, Kaswan R.L, Sundberg J.P. (1987) Squamous cell carcinoma of the corneoscleral limbus in a dog. JAVMA ;190(11):1430-1432.

Leib M.S., Saunders G.K. and M.J., Dallman(1986) Squamous cell carcinoma of the prostate gland in a dog. JAAHA ;22:509-514.

Li G and E.M.,Sturgis. (2006) The role of human papillomavirus in squamous cell carcinoma of the head and neck. Curr Oncol Rep ;8(2):130-139.

McEntee M.C., Page R.L. and A. Theon et al., (2004) Malignant tumor formation in dogs previously irradiated for acanthomatous epulis. Vet Radiol Ultrasound ;45(4):357-361.

O'Brien M.G, Berg .J andS.J. Engler.(2016) Treatment by digital amputation of subungual squamous cell carcinoma in dogs: 21 cases. JAVMA ;201(5):759-761.

Plassmann, P. (2005). Measuring wounds. J. Wound. Care. 4(6): 269-72.

Porat, S.R., Roussa , M. and S.Shosan. (2008). Improvement of gliding functions of flexor tendons by topically applied enriched collagen solution. J. Bone. and Joint. Surg. 62:208.

Schmidt B.R., Glickman N.W. and D.B.DeNicola (2001) Evaluation of piroxicam for the treatment of oral squamous cell carcinoma in dogs. JAVMA ;218(11):1783-1786.

Ward D.A., Latimer K.S. and R.M. Askren (2007) Squamous cell carcinoma of the corneoscleral limbus in a dog. JAVMA;190(11):1430-1432.

\section{How to cite this article:}

Gokulakrishnan. M, L. Nagarajan, Ramani and Sowmiya. 2020. Surgical Management of Periorbital Squamous Cell Carcinoma through Wide Margin Excision Followed by Transposition Skin Flap in a Dog. Int.J.Curr.Microbiol.App.Sci. 9(02): 2489-2492. doi: https://doi.org/10.20546/ijcmas.2020.902.282 